\title{
Mejora de las condiciones higiénico-sanitarias, de la salud y ambientales de los niños en edad escolar de las comunidades Gatazo - Colta \\ Improvement of hygienic-sanitary, health and environmental conditions of school-age children form Gatazo communities - Colta
}

\author{
LÓPEZ PUMALEMA JOSÉ ISRAEL, BASANTES AVALOS JENNY LILIAN* \\ Maestría en Gestión de Proyectos de Desarrollo \\ Instituto de Posgrados y Educación Contínua, Escuela Politécnica del Chimborazo, Panamericana Sur km 1 1/2, Riobamba, Ecuador. \\ *iebasantes@espoch.edu.ec
}

\section{RESUMEN:}

La presente investigación tiene su base en la carencia de un método de evaluación de impacto para el proyecto de vinculación con la sociedad: "Mejora de las condiciones higiénico-sanitarias, de salud y ambientales de los niños en edad escolar de las comunidades Gatazo - Colta". Para lo cual se emplea un método cuantitativo no experimental conocido como modelo antes-después que compara la información de línea base con la información actual recopilada por el investigador a través de una encuesta y la realización de análisis de laboratorio coproparasitarios. Para ello, se establecieron pruebas de hipótesis de medias y proporciones a un 95\% de nivel de confianza utilizando el software SPSS. Se complementa con un método cualitativo mediante entrevistas a los beneficiarios y otros actores relevantes del proyecto. De esta manera, se determinó que el nivel de desnutrición varió del $1 \%$ al $8 \%$ en los niños en edad escolar y dado que, el estado nutricional cambiaba significativamente en el tiempo, se concluyó que desnutrición aumentó. En lo referente a los hábitos alimenticios se reflejó que el cambio de dieta y hábitos alimenticios significativo. Sin embargo, debido que no se realizaron las actividades correspondientes estos resultados no se pueden atribuir al proyecto. Enfermedades como la diarrea pueden manifestarse por la presencia de parásitos algunos de ellos asociados al consumo de agua no clorada o la falta de higiene. De forma general, se concluyó que el proyecto de vinculación cumplió parcialmente con los objetivos que se plantearon pues se finalizaron el $55,56 \%$ y los resultados esperados no fueron alcanzados.

Palabras Clave: Evaluación de impacto, método no experimental, modelo antes-después, proyecto de vinculación con la sociedad. 


\section{INTRODUCCIÓN}

La evaluación de impacto es una aproximación que mide el resultado de la intervención de un programa aislando otros posibles factores [1]. Existen tres principales razones para evaluar. La primera es la necesidad de evidenciar qué funciona y qué no funciona. La segunda está relacionada con la implementación de mejoras a través de programas o políticas. Y la tercera se asocia a la información como clave de la sustentabilidad. En tal virtud, realizar una evaluación de impacto implica probar si la acción realizada ha cumplido su propósito en la población en la que se aplicó.

En el caso actual, es conocer si se cumplieron o no los objetivos del proyecto de vinculación aplicado en las comunidades de Colta y Gatazo, específicamente de las unidades educativas 15 de Agosto y UNICEF. Esto quiere decir, saber si se realizó el levantamiento de la línea base, si la desnutrición en los niños se redujo, si se promulgó una adecuada alimentación, si se disminuyeron las diarreas o los síntomas de anemia y cefaleas, si se incrementaron los controles médicos o si se evaluaron los logros alcanzados.

Evaluar un proyecto de vinculación es medir si las universidades y escuelas politécnicas garantizan el derecho a la educación superior y se encuentran enlazadas a la demanda académica para cumplir con el principio de pertinencia. El mismo que "consiste en que la educación superior responda a las expectativas y necesidades de la sociedad" [2]. análisis descriptivo y evaluación ex post. Sección V Conclusiones del estudio realizado.

\section{DEFINICIONES DE EVALUACIÓN}

Los proyectos sociales constituyen la unidad más pequeña a la cual se puede asignar recursos para aplicar procesos y actividades que cambien una realidad. El propósito de los proyectos sociales es la disminución, eliminación o solución a un problema, cumpliendo para ello con algunas condiciones: La definición de la problemática de forma cuantitativa antes de empezar el proyecto. Contar con objetivos claros y precisos para posteriormente poder someterlos a evaluación. Identificar el público objetivo, así como también su localización en el espacio. Establecer fechas de inicio y fin del proyecto [3].

Un proyecto social constituye un motivo perfecto y acertado para identificar el nivel de participación de una sociedad, pudiendo realizar un estudio analítico de un grupo objetivo [4]. Estos proyectos poseen recursos propios, que pueden ser transferidos directa o indirectamente al grupo objetivo. Generalmente, estos proyectos están relacionados con las políticas de un país. De esta manera, se ampara la generación de programas que, a su vez, engloban proyectos asociados a combatir una problemática social. Esta problemática generalmente está relacionada con la pobreza. Por ello, se puede asumir que los proyectos sociales se emplean con mayor frecuencia en lugares o sectores más necesitados [3]. Para ello, se define la población de interés o universo es aquel conjunto de unidades elegidas para participar del tratamiento planteado en el proyecto [5]

Evaluar es examinar sistemática y objetivamente el diseño, la puesta en marcha y los resultados obtenidos o que quieren obtener al aplicar

Este artículo está organizado de la siguiente manera: Sección Il presenta
una descripción de las principales definiciones de evaluación, los tipos
y métodos de utilizados para evaluar en los proyectos sociales. Sección
III Metodología de la presente investigación. Sección IV Resultados del

Este artículo está organizado de la siguiente manera: Sección II presenta
una descripción de las principales definiciones de evaluación, los tipos
y métodos de utilizados para evaluar en los proyectos sociales. Sección
III Metodología de la presente investigación. Sección IV Resultados del 
programa, proyecto o política [6]. Una evaluación se considera completa cuando está integrada por cuatro elementos: monitoreo, evaluación del proceso, evaluación costo-beneficio y evaluación de impacto. El monitoreo se relaciona con la visualización de que lo planificado se esté implementando, con ello es factible detectar a tiempo posibles inconvenientes en la ejecución. La evaluación del proceso se centra en el funcionamiento del programa y los problemas en la prestación de servicios. La evaluación costo-beneficio o costoefectividad determina monetaria o no monetariamente otras posibles formas de emplear estos recursos en otras actividades. Finalmente, la evaluación de impacto es un tipo de evaluación sumativa pues se realiza al culminar la aplicación de un programa o proyecto [7]. De esta manera, permite conocer si se alcanzaron los efectos deseados en la población en la que se dedicó la intervención; incluso puede analizar efectos no contemplados en dicha intervención [1].

\section{A.-Tipos de Evaluación}

Según la temporalidad. - Desde el punto de vista económico se considera el tiempo en el que se ejecuta la evaluación. Por ello, la evaluación de impacto se puede realizar ex ante o ex post a la aplicación del programa o política. Por un lado, la evaluación ex ante o estructural usualmente se relaciona con modelos estructurales de comportamiento estimados en base a datos y según supuestos propios del modelo. En este tipo de evaluación se puede evaluar el posible efecto del programa o política antes de ser aplicado; por ejemplo, a través de una micro-simulación. La evaluación ex ante es una aproximación más tradicional basada en el estándar de racionalidad económica. Por otro lado, la evaluación ex post o forma reducida se aplica posteriormente a la aplicación del programa o política. Esta evaluación puede ser utilizada para inferir información sobre el comportamiento; si existiera suficiente validez, esta información puede servir para predecir el efecto de una futura política o programa. Sin embargo, estas evaluaciones son complementarias debido a que los resultados empíricos son necesarios para calibrar los modelos teóricos y a la inversa, los modelos teóricos son útiles para interpretar los resultados empíricos y determinar la su potencial validez [1]

Según quién realiza la evaluación. - Se distinguen cuatro tipos de evaluación de acuerdo con quién la realiza: externa, interna, mixta y participativa. La evaluación externa es aquella en la que los evaluadores nunca han actuado en el proyecto previamente y poseen experiencia en evaluación de proyectos o son eruditos en áreas afines al mismo. Por otro lado, la evaluación interna aísla este inconveniente pues son las personas que actuaron en el proyecto quienes se encargarán de realizar la evaluación. La evaluación mixta que no es más que una fusión de las dos evaluaciones antes mencionadas, es decir, el evaluador externo y el evaluador interno trabajan de forma conjunta y de esta manera se minimizan las desventajas individuales de dichas evaluaciones sin considerar a la población objetivo. La evaluación participativa reduce la brecha entre evaluador y beneficiario está dirigida a proyectos pequeños y de preferencia sociales cuyo éxito radica en la participación dinámica de los beneficiarios, inclusive desde el diseño hasta la evaluación del proyecto. [8].

Según la escala del proyecto. - Al considerar el número de beneficiarios y los recursos se pueden distinguir la evaluación de proyectos grandes y de proyectos pequeños. Dentro de estos tipos de evaluación se diferencian algunos parámetros como: estrategia de evaluación, lógica de evaluación, diseño de evaluación, técnicas de análisis, resultados de la evaluación y los evaluadores. 
Según los destinatarios. - El enfoque de la evaluación está relacionado con el para qué y para quién se evalúa. El para qué de la evaluación permite diferenciar proyectos o alternativas para el mismo proyecto cuya finalidad sea incrementar la eficiencia y eficacia de estos. De acuerdo con el "para quién" se plantea el diseño, contenido, metodología y tipo de análisis. Con ello, se distinguen tres tipos de destinatarios: directivos superiores, administradores y técnicos. Los directivos superiores que son los encargados de establecer políticas, prioridades y asignación de recursos. Los administradores optimizan los insumos y productos para alcanzar los objetivos planteados. Finalmente, los técnicos son los ejecutores, quienes operativizan y ponen en práctica el proyecto y los modelos [8].

\section{B.- Métodos de Evaluación}

Dentro de los métodos de evaluación de impacto se encuentran los métodos cuantitativos y los métodos cualitativos. Para determinar la metodología que se va a aplicar es indispensable establecer le grupo contrafactual y en el caso de que no existiere como tal se debe plantear un alternativo [1]. El contrafactual responde a la pregunta "żsi el proyecto no se hubiese ejecutado la situación actual sería la misma?" [9], es decir, se requiere aislar el efecto de la aplicación del proyecto [1]. Un escenario contrafactual marca el diseño de la evaluación, estos diseños se establecen en tres grandes grupos: los experimentales, los cuasi experimentales y los no experimentales [3]. Los diseños experimentales están relacionados a la aleatoriedad y los cuasi experimentales y los no experimentales a la no aleatoriedad.
Los diseños experimentales o de aleatorización resultan metodologías sólidas. La aleatoriedad al momento de la asignación de los beneficiarios del proyecto permite identificar el grupo de tratamiento y el de control con características comparables. Esto proporciona inclusive la determinación de la muestra que conforma los grupos mencionados. De esta manera, el grupo de control es posible que se etiquete como el contrafactual. Lo que a su vez simplifica la interpretación de los resultados. Sin embargo, este tipo de diseño conlleva algunas desventajas. Existe un riesgo de bajo nivel ético en la asignación de los beneficiarios o la intrusión de cuestiones políticas. En el caso de proyectos de largo alcance, se pueden anular los grupos de control debido a que los grupos de tratamiento serían todos los individuos inmersos en la política aplicada. Puede surgir una complicación en los individuos del grupo de control debido los cambios de rasgos que pueden sufrir durante la ejecución del proyecto. Lo que provocaría la tergiversación de la comparación del grupo de control con el grupo de tratamiento. Finalmente, puede resultar complejo el cumplir con una verdadera asignación aleatoria [1].

Por otro lado, se encuentran los diseños cuasi experimentales. Este tipo de método se emplea por la falta de especificación de los grupos de tratamiento y control para la aplicación de un diseño experimental. En este caso, el grupo de control se establece mediante técnicas econométricas que involucran un control estadístico riguroso. Estas técnicas pueden ser: métodos de pareo o controles construidos, doble diferencia o diferencia en diferencias, variables instrumentales 0 control estadístico y comparaciones reflexivas. También es factible la creación de un grupo de control no aleatorio antes de la aplicación del proyecto [1] 
A diferencia de los métodos experimentales y cuasi-experimentales, los métodos no experimentales no pueden utilizar un grupo de control para analizar el impacto de un proyecto. Estos métodos utilizan a la población objetivo para realizar dicha aproximación. Lo que implica que la comparación con el grupo testigo se imposibilite, descartando los efectos de variables exógenas. Entre los diseños no experimentales se encuentran: modelo antes-después, modelos solo después con grupo de comparación y modelo solo después.

\section{Elección e Implementación}

Lo más recomendable para una eficaz evaluación de impacto es combinar los métodos cuantitativos y los métodos cualitativos. Con ello, se observa la parte cuantificable del programa y se complementa con los procesos e institucionalidad generados a partir del mismo. A esto, se puede acoger una perspectiva que abarque la aplicación o no aplicación del programa, también el antes y el después de dicha aplicación que contemple datos básicos y de seguimiento. En términos generales, una evaluación de impacto óptima contendrá: un contrafactual que contenga un grupo de tratamiento y un grupo de control a través de un diseño experimental o cuasiexperimental, considerar el antes y el después del accionar del programa tomando en cuenta el tiempo transcurrido, un tamaño significativo de los grupos de control y tratamiento para obtener estadísticas robustas, añadir el análisis de costos-beneficios o eficacia e incluir métodos cualitativos para la triangulación del análisis de los resultados [1].

Implementar una evaluación de impacto contempla un nivel de complejidad y un costo, lo que conlleva a formular una solución para una serie de inconvenientes. Estas dificultades se pueden minimizar cuando la evaluación es diseñada al inicio del programa. De esta manera, se obtendrían mejores resultados e incluso se pueden tomar acciones correctivas oportunas [1]. Existen algunas características principales que deben cumplir los proyectos para que sean considerados para una evaluación de impacto. En primera instancia se debe contemplar el nivel de innovación e importancia que tiene el proyecto. Como segundo punto es conocer si el proyecto está diseñado para ser replicado. En tercer lugar, se debe considerar la etapa en la que el proyecto se encuentra, es decir, debe tener un tiempo de ejecución que permita establecer una evaluación.

\section{METODOLOGÍA DE LA INVESTIGACIÓN}

\section{Metodología y tipos de investigación}

El presente estudio se realizó en la localidad de Gatazo, ubicado en el cantón Colta. El diseño de esta investigación se clasifica como un diseño cuantitativo no experimental del tipo longitudinal de evolución, debido a que, se realiza una comparación entre la línea base levantada y la información actual recopilada sobre los elementos del proyecto de vinculación con la sociedad "Mejora de las condiciones higiénicosanitarias, de salud y ambientales de los niños en edad escolar de las comunidades Gatazo - Colta". Este cotejo se efectúa para cumplir con el principal objetivo de este análisis: la evaluación ex post del proyecto en mención.

Dentro de los tipos de investigación que alcanza este estudio, se contemplan el descriptivo y el correlacional. Se considera una investigación descriptiva puesto que, se requiere conocer a detalle información referente a la desnutrición, alimentación, enfermedades y controles médicos de la población objetivo de Gatazo tanto en su línea base como en la actualidad. Y es que la investigación descriptiva especifica de manera precisa e independiente la medida de cada una de las características de los individuos o comunidades en análisis [10].

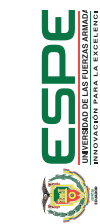


Se aplica la investigación correlacional pues se requiere conocer si existe algún efecto con la implementación del proyecto de vinculación. Por definición, la investigación correlacional tiene como objetivo señalar el grado de relación entre dos o más variables que puede ser positiva o negativa. Además, si en este análisis se incorporan múltiples variables, es factible emitir una explicación más completa de la situación en estudio [10].

\section{Métodos de Investigación}

Para llevar a cabo la presente investigación fue necesario plantear métodos teóricos y empíricos. Dentro de los métodos teóricos se encuentran: analítico-sintético e inductivo-deductivo [1 1]. Mientras que como métodos empíricos se tomó en consideración el empíricoanalítico.

EL método analítico-sintético se basa en procesos inversos, pero a su vez complementarios, que son: el análisis y la síntesis. En este sentido, se realizará la parte analítica a través de la aplicación de herramientas estadísticas descriptivas e inferenciales para determinar el efecto negativo o positivo de cada una de las variables contempladas en el proyecto de vinculación. Con ello, es posible sintetizar el impacto general del proyecto en la comunidad en la que se aplicó dicho proyecto, en este caso, la población de Gatazo del cantón Colta.

De manera similar, el método inductivo - deductivo, se forma de dos corrientes opuestas: la inducción y la deducción. En este caso, la inducción estará direccionada al análisis de ámbitos como la desnutrición o el tipo de alimentación de la población. Su finalidad será: conocer si estos aspectos mejoraron o no a partir de la puesta en marcha del proyecto sobre mejorar las condiciones de vida del grupo en análisis, que se instauraría como el rasgo en común. Se observarán a priori y posteriori estos parámetros, cuya hipótesis estará direccionada al cumplimiento de los objetivos del proyecto implantado, se verificará a través de herramientas estadísticas inferenciales para concluir con el conocimiento del grado de afección resultante de la implementación de dicho proyecto. En el caso de la deducción, se partirá de la generalidad del accionar del proyecto de vinculación para determinar su actuar en el territorio. Es decir, disgregar cada uno de los puntos de acción que buscaba el proyecto y su nivel de afección. Nuevamente, al empatar la inducción y la deducción es posible conocer si el proyecto en estudio cumplió o no con los objetivos planteados.

En el método empírico-analítico, la objetividad muestra un papel preponderante ya que existe la facilidad de réplica de la investigación, contemplada en la etapa experimental. Por ello, la presente evaluación de impacto se aplicará con un método de análisis entre la línea base y la situación actual de la población objetivo. Este método se conoce como modelo antes-después. En este caso en particular, el antes será la línea base y el después se constituirá por la información recopilada en campo. A partir de este análisis es plausible evidenciar la existencia o no de una variación de los aspectos de la comunidad de Gatazo en lo posterior a la aplicación del proyecto de vinculación.

\section{Enfoque y alcance de la investigación}

El enfoque de esta investigación es principalmente de tipo cuantitativo y se complementa con una parte cualitativa. Debido a la diversidad de herramientas que se pueden utilizar para realizar evaluación de impacto y combinada con la complejidad del análisis de la ejecución de proyectos sociales, el evaluador está en la potestad de adoptar varios métodos de investigación [7]. Por ello, el alcance del presente estudio 
considera en primera instancia el tipo de investigación a aplicarse en el será descriptivo y correlacional puesto que debe responder a las necesidades propias de la evaluación de resultados. Esto involucra el conocimiento de los efectos a corto y mediano plazo de los proyectos sociales. Lo que implica que se aplique la evaluación más usada para estos casos: la evaluación de impacto.

En este sentido, la presente evaluación de impacto se puede tipificar según la conceptualización de tipo de evaluación en una evaluación ex-post externa de un proyecto pequeño dirigida a directivos superiores. Esta caracterización se debe a que es realizada posteriormente a la ejecución del proyecto por un agente externo, en este caso un egresado de maestría.

Además, se considera un proyecto pequeño debido al bajo número de beneficiarios y el poco presupuesto asignado. La presente evaluación de impacto permitirá que directivos encargados de la vinculación con la sociedad en la ESPOCH y docentes que participan en dichos proyectos tomen acciones preventivas y de fortalecimiento, según convenga, a futuros proyectos de vinculación con la sociedad.

El método a utilizarse será un método cuantitativo no experimental debido a la falta de un grupo de control o de comparación con el cual se puede contraponer el grupo de tratamiento. El modelo específico será el denominado "antes-después" en el que se comparará la línea base del proyecto con la nueva información recopilada. Se complementa con un método cualitativo rural rápido. Esto permitirá cumplir el principal objetivo de esta investigación. Para comprender si la diferencia de las variables en estos dos puntos en el tiempo es significativa se aplicarán pruebas de hipótesis para medias y proporciones al $95 \%$ de confianza.

\section{Muestra}

Considerando que, la población o universo muestral es un conjunto de seres animados o inanimados cuyas características constituyen una fuente de información [12] Adicionalmente, al establecer el modelo antes-después como un método no experimental para evaluación de impacto. La población de estudio es la misma población que conforma el grupo de tratamiento al que se le aplicó el proyecto. Además, dado que han transcurrido dos años, varios niños se encontrarán cursando el bachillerato o habrán dejado de estudiar. Por lo cual, la población del presente estudio contempla únicamente a los niños que se encuentran aún en edad escolar en las escuelas: 15 de Agosto y UNICEF. Ambas escuelas localizadas en comunidad de Gatazo en el cantón Colta. La población está conformada por 90 niños y niñas, que se encuentran distribuidos de la siguiente manera: 64 niños en la escuela 15 de Agosto y 26 niños en la escuela UNICEF.

La unidad de análisis son los niños que fueron partícipes del inicio de la aplicación del proyecto de vinculación de las escuelas: 15 de Agosto y UNICEF de la comunidad de Gatazo en el cantón Colta. De acuerdo con esto y sabiendo que la muestra es un subconjunto seleccionado que representa a la población [12]. Para establecerla, se aplicó un muestreo aleatorio simple con un $95 \%$ de confianza. Se considera la estimación de la proporción poblacional que generalmente es aplicada en investigación [12]:

$$
n=\frac{Z^{2} p q N}{N E^{2}+Z^{2} p q}
$$

Donde,

$\mathrm{N}$ : tamaño de la población

$\mathrm{n}$ : tamaño de la muestra 


\section{Z: nivel de confianza}

p: variabilidad positiva

q: variabilidad negativa

E: precisión o error

Considerando que la población es de 90 niños, el nivel de confianza del 95\% lo que implica que el valor de Z es de 1.96, una variabilidad positiva y negativa del $5 \%$ y un error del $5 \%$; se tiene que, el tamaño de la muestra se estableció en 73 niños.

\section{Técnicas e instrumentos de recolección de información}

La información primaria es recopilada directamente por el investigador en una relación expresa con el sujeto de estudio. Para obtener información primaria se utilizó encuestas y entrevistas para obtener la información actual de los individuos en estudio. La encuesta se elaboró en base a la planteada para obtener la línea base a razón de darle comparabilidad en la información. Por otro lado, las entrevistas se las realizó principalmente a autoridades que participaron en la implementación del proyecto de vinculación de las unidades educativas en mención.

La información secundaria es compilada por el investigador de trabajos realizados previamente, este tipo de información no requiere contacto directo con el sujeto de estudio. Dentro de la información secundaria se indagó en informes realizados por parte de los docentes que intervinieron
Para ejecutar la evaluación ex post, se requiere información que contenga el entorno previo y posterior a la ejecución del proyecto

de vinculación. La información con la que cuenta la investigación se en el diseño y ejecución del proyecto de vinculación y que, a su vez, fueron entregados a la Dirección de Vinculación de la ESPOCH. enfoca en la línea base levantada. Esta línea base abarca datos de talla y peso que permiten obtener las curvas de desnutrición. También contiene información relacionada a hábitos de consumo de alimentos y agua y, uso y tipo de servicio higiénico. Adicionalmente, se encuentran disponibles los resultados de los exámenes coproparasitarios aplicados a los niños. Sin embargo, no se cuenta con información posterior. Por lo que, es necesario aplicar nuevamente los exámenes coproparasitarios, encuestas, entrevistas y tomar las medidas de talla y peso de los niños. Con ello, se puede plantear el escenario posterior a la aplicación del proyecto que facilita la comparación con la línea base.

\section{Método de evaluación seleccionado}

Para la evaluación de impacto ex post, se escogió el método antes después, un método cuantitativo no experimental que no considera un grupo de control para realizar la comparación. Este método utiliza la información de línea base levantada como parte de la ejecución del proyecto y la nueva información levantada sobre la población objetivo para realizar la comparación entre dos puntos en el tiempo. Para saber si la diferencia existente entre la comparación y la línea base es significativa se aplican pruebas de hipótesis para medias y proporciones, según sea el caso. Se complementó con un método cualitativo rural rápido que recopila información de directivos y actores beneficiarios del proyecto a través de entrevistas.

Estas pruebas permiten probar afirmaciones sobre características de interés para lo cual se plantean dos hipótesis: la hipótesis nula o inicial $\left(H_{0}\right)$ representando el suceso en análisis y la hipótesis alternativa $\left(H_{1}\right)$ que simboliza el cambio relevante que surge cuando se altera el curso normal del evento. Además de las hipótesis, se requiere establecer un estadístico de prueba que facilitará la decisión entre aceptar o rechazar 
la hipótesis nula para descartar o afirmar la hipótesis alternativa. La distribución de este estadístico debe ser conocida. Este estadístico marca la región de rechazo o crítica de la prueba para descartar o confirmar la hipótesis nula [12]. A continuación, se describe tanto la prueba de hipótesis para medias como para proporciones que se utilizará en esta sección.

Prueba de hipótesis para diferencia de medias:

1. Hipótesis nula. $H_{0}: \mu_{L B}-\mu_{L C}=0$

2. Hipótesis alternativa. $H_{1}: \mu_{L B}-\mu_{L C} \neq 0$

3. Estadístico de prueba. $t_{o b s}=\frac{\left(\bar{x}_{L B}-\bar{x}_{L C}\right)}{\sqrt{\frac{\sigma_{L B}^{L C}}{n_{L B}}+\frac{\sigma_{L C}^{L C}}{n_{L C}}}}$

4. Región de rechazo. $t_{o b s}<-t_{\alpha / 2}$ ó $t_{o b s}>t_{\alpha / 2}$

\section{Donde,}

$\mu_{L B}:$ media en la línea base

$\mu_{L C}:$ media en la línea de comparación

$t_{o b s}$ : estadístico de prueba observado que sigue una distribución determinada

$\bar{x}_{L B}:$ media muestral en la línea base

$\bar{x}_{L C}:$ media muestral en la línea de comparación

$n_{L B}:$ tamaño muestral en la línea base

$n_{L C}$ : tamaño muestral en la línea de comparación

$\sigma$ : desviación estándar

$\alpha$ : nivel de significación

$t_{\alpha / 2}$ : estadístico de prueba de la distribución

Prueba de hipótesis para diferencia de proporciones:

1. Hipótesis nula. $H_{0}: p_{L B}-p_{L C}=0$

2. Hipótesis alternativa. $H_{1}: p_{L B}-p_{L C} \neq 0$
3. Estadístico de prueba. $\chi^{2}{ }_{o b s}=\frac{\hat{p}_{L B}-\hat{p}_{L C}}{\sqrt{\hat{p} \widehat{q}\left(\frac{1}{n_{L B}}+\frac{1}{n_{L C}}\right)}}$

4. Región de rechazo. $\chi^{2}{ }_{o b s}<\chi_{\alpha / 2}^{2}$ ó $\chi^{2}{ }_{o b s}>\chi_{\alpha / 2}^{2}$

Donde,

$p_{L B}$ : proporción en la línea base

$p_{L C}$ : proporción en la línea de comparación

$\chi_{o b s}^{2}$ : estadístico de prueba observado que sigue una distribuci determinada

$n_{L B}$ : tamaño muestral en la línea base

$n_{L C}:$ tamaño muestral en la línea de comparación

$\hat{p}$ : proporción muestral

$\hat{q}$ : proporción muestral $(1-\hat{p})$

$\alpha$ : nivel de significación

$\chi_{\alpha / 2}^{2}$ : estadístico de prueba de la distribución

Para aceptar o rechazar la hipótesis nula $\left(H_{0}\right)$ es necesario referirse al nivel de significación observado o p-valor. Este valor es el mínimo al cual la hipótesis nula es rechazada de acuerdo con el nivel de significación. Si el valor de $p$ es menor al nivel de significación $(p<a)$, la hipótesis nula $\left(H_{0}\right)$ se rechaza, aceptándose automáticamente la hipótesis alternativa $\left(\mathrm{H}_{1}\right)$.

\section{RESULTADOS Y DISCUSIÓN}

Desnutrición

Para el cálculo de la desnutrición, se toma en cuenta al índice de masa corporal ya que, es el índice más utilizado para determinar signos de alerta y diagnosticar desnutrición. Para lo cual, se tomará como fuente las mediciones realizadas de talla y peso. Antes de entrar en el análisis 
del índice de masa corporal, se estudiará un análisis individual del comportamiento de la talla y peso.

En la línea base, el peso promedio alcanza un valor de $26,86 \mathrm{~kg}$ mientras que en la línea de comparación llega a 29,07 kg. No existe mayor diferencia en la línea base con respecto al sexo; puesto que, el peso medio en los niños es de 26,69 y en las niñas 26,91 . En tanto, en la línea de comparación la diferencia es un poco más marcada, los niños tienen un peso promedio de 29,93 kg mientras las niñas poseen un peso de $28,58 \mathrm{~kg}$ en promedio.

Por otro lado, a nivel de línea base, la talla media se ubica en 115,94 cm; mientras tanto, en la línea de comparación se ubica en 128,24 $\mathrm{cm}$. Con respecto al sexo, los niños en la línea base muestran una mayor talla promedio $(119,56 \mathrm{~cm})$ que las niñas $(113.54 \mathrm{~cm})$. Esta relación se mantiene en la línea de comparación, los niños consiguen una media de la talla de 131,04 cm y las niñas de 126,67 cm.

La diferencia de medias obtenida para el peso y la talla se ha obtenido a un nivel de significación del $5 \%$. En el caso del peso, la diferencia es de 6,81 y es significativa. Al analizar la talla, existe una diferencia de 11,89; también significativa.

Por otro lado, en la Tabla I se muestra la prueba chi-cuadrado como resultado de analizar el estado nutricional en ambas líneas. La hipótesis nula señala que el estado nutricional de la línea base no está asociado con la línea de comparación. En tanto, la hipótesis nula refleja una asociación entre estas variables, es decir, el estado nutricional sí ha cambiado significativamente entre la línea base y la línea de comparación. Dado que el p-valor de esta prueba es de 0,018 menor que el nivel de significancia del 5\%, rechazamos la hipótesis nula y aceptamos la hipótesis alternativa.

\section{Tabla 1}

Relación del estado nutricional con el tipo de año

\begin{tabular}{|l|r|r|r|}
\hline \multicolumn{4}{|c|}{ PRUEBAS DE CHI-CUADRADO } \\
\hline & \multicolumn{1}{|c|}{ Valor } & \multicolumn{1}{|c|}{ gl } & \multicolumn{1}{c|}{$\begin{array}{c}\text { Sig. asintótica } \\
\text { (bilateral) }\end{array}$} \\
\hline Chi-cuadrado de Pearson & $11,884^{\circ}$ & 4 & 0,018 \\
\hline Razón de verosimilitudes & 13,429 & 4 & 0,009 \\
\hline $\begin{array}{l}\text { Asociación lineal por } \\
\text { lineal }\end{array}$ & 0,597 & 1 & 0,440 \\
\hline N de casos válidos & 163,000 & & \\
\hline
\end{tabular}

Fuente: Información de peso y talla de la línea base del proyecto y del nuevo levantamiento de datos

Con ello, se evidencia el cambio significativo de talla y peso. Además, existe una variación significativa del estado nutricional. Sin embargo, no se puede afirmar una disminución en la desnutrición. En primer lugar, no existe un alto nivel de desnutrición en la línea base (1\%). En segundo lugar, la desnutrición se incrementa al $8 \%$ en la línea de comparación. Y finalmente, en tercer lugar, la diferencia es significativa en el estado nutricional. Por lo que, se puede concluir que la desnutrición aumentó significativamente.

\section{ALIMENTACIÓN ADECUADA}

Para la alimentación adecuada, se consideran algunos aspectos como el tipo de alimentos que consumen en el hogar las familias de los niños en edad escolar. 
Se observa la proporción de consumo de diferentes alimentos distribuidos en la línea base y en la línea de comparación. La mayor parte de los alimentos tiene un consumo moderado en la línea base, incluso se incrementa al realizar la observación de la línea de comparación. Además, se evidencia un incremento en el consumo de vegetales y frutas tanto de la sierra como de la costa.

En la Tabla II se muestra la existencia de diferencias significativas en casi todos los alimentos consultados. Los más significativos son los vegetales, las frutas de la sierra, las frutas de la costa, las carnes blancas y las carnes rojas, con un p-valor de cero. En tantos las grasas saturadas y la sal también marcan un consumo significativo, pero con p-valores de 0,010 y 0,007; respectivamente. Los p-valores son significativos al $95 \%$.

Tabla 2

Diferencias del consumo de alimentos

\begin{tabular}{|c|c|c|c|c|c|c|c|}
\hline & \multicolumn{7}{|c|}{ Prueba T para la igualdad de medias } \\
\hline & \multirow{2}{*}{$t$} & \multirow[b]{2}{*}{ gl } & \multirow{2}{*}{$\begin{array}{l}\text { Sig. } \\
\text { (bilateral) }\end{array}$} & \multirow{2}{*}{$\begin{array}{l}\text { Diferencia } \\
\text { de medias }\end{array}$} & \multirow{2}{*}{$\begin{array}{l}\text { Error típ. de } \\
\text { la diferencia }\end{array}$} & \multicolumn{2}{|c|}{$\begin{array}{c}95 \% \text { Intervalo de } \\
\text { confianza para la } \\
\text { diferencia } \\
\end{array}$} \\
\hline & & & & & & Inferior & Superior \\
\hline Sal & $-2,763$ & 102 & 0,007 & $-0,365$ & 0,132 & $-0,628$ & $-0,103$ \\
\hline Azúcar & $-2,106$ & 102 & 0,038 & $-0,288$ & 0,137 & $-0,560$ & $-0,017$ \\
\hline $\begin{array}{l}\text { Grasas } \\
\text { saturadas }\end{array}$ & $-2,609$ & 101 & 0,010 & $-0,419$ & 0,160 & $-0,737$ & $-0,100$ \\
\hline $\begin{array}{l}\text { Grasas } \\
\text { insaturadas }\end{array}$ & $-1,511$ & 99 & 0,134 & $-0,213$ & 0,141 & $-0,493$ & 0,067 \\
\hline Vegetales & 6,132 & 81 & 0,000 & 1,035 & 0,169 & 0,699 & 1,371 \\
\hline $\begin{array}{l}\text { Frutas de la } \\
\text { sierra }\end{array}$ & 8,506 & 97 & 0,000 & 1,152 & 0,135 & 0,883 & 1,421 \\
\hline $\begin{array}{l}\text { Frutas de la } \\
\text { costa }\end{array}$ & 6,856 & 95 & 0,000 & 1,028 & 0,150 & 0,730 & 1,325 \\
\hline $\begin{array}{l}\text { Carnes } \\
\text { blancas }\end{array}$ & 5,825 & 97 & 0,000 & 0,918 & 0,158 & 0,605 & 1,231 \\
\hline Carnes rojas & 3,733 & 100 & 0,000 & 0,639 & 0,171 & 0,299 & 0,979 \\
\hline
\end{tabular}

Fuente: Información de encuestas de la línea base del proyecto y del nuevo levantamiento de datos
En primera instancia, la diferencia de consumo de tipo de alimentos prevé un cambio en los hábitos alimenticios de la población objetivo. Sin embargo, el consumo de frutas y hortalizas no presenta cambio alguno. Se debe agregar a este análisis que las actividades relacionadas con la concientización y el cambio de dietas no se realizó. Por tanto, la variación presente en el tipo de alimentos que consumen las familias se puede deber a otro tipo de factores que no estaban contemplados en el proyecto.

\section{CONTROLES MÉDICOS}

En la Tabla III se reflejan los principales resultados de los exámenes coproparasitarios en la línea base y en la línea de comparación. Por esto, los siguientes párrafos describirán a detalle cada uno de los resultados y su asociación a diversas enfermedades que podrían padecer los niños.

Como primer resultado representativo se encuentra el moco que está directamente relacionado con enfermedades gastrointestinales. Se puede observar la presencia de moco $(++)$ con un $66,7 \%$ en la línea base; sin embargo, en la línea de comparación este desaparece. El moco (+) alcanza un $33,3 \%$ en la línea base y un $100 \%$ en la línea de comparación. Es decir que, de cierta manera en la línea de comparación se ha reducido la presencia de enfermedades gastrointestinales.

Por otro lado, el quiste ameba coli (+) aparece con un $97,4 \%$ en la línea base, pero se reduce en la línea de comparación a $66,7 \%$. Sin embargo, el quiste ameba coli $(++)$ en la línea base corresponde al $2,6 \%$ y se incrementa al $33,3 \%$ en la comparación. Lo que implica que, el quiste ameba coli en los niños se ha agudizado. Por sí solo, 
este tipo de quiste no provoca mayores afecciones en la salud de los niños. Sin embargo, al analizarlo con el estado nutricional, en especial con el bajo peso y la desnutrición, se puede evidenciar que en la línea base el porcentaje de niños que presentaron ameba coli y bajo peso fue de 19,4\%. Este porcentaje disminuye en la línea de comparación a 5\% pero aumenta la relación con la desnutrición a un $10 \%$ cuando en la línea base era nula esta correspondencia. Lo que implicaría aparentemente una desmejora en la salud de los niños después del proyecto. En el caso del quiste ameba histolytica que es un microorganismo que se desarrolla en aguas contaminadas o poco cloradas, también está asociada a la falta de higiene de las manos; así como de los alimentos destinados al consumo. En la línea base alcanza el $100 \%$ en (+). En tanto, en la línea de comparación este porcentaje se reduce a $78,6 \%$ pero se incrementa en $(++)$ al $21,4 \%$. Esto estaría relacionado con la falta de tratamiento adecuado del agua para el consumo humano o también a la falta de buenas prácticas de higiene tanto personal como de los alimentos que consumen.

Entre los resultados, también se encontró blastocystis hominis únicamente en la línea de comparación con un 100\%. La presencia de este microorganismo no determina algún comportamiento en particular. Solamente si se vincula a que quien lo porte sea susceptible a contraer enfermedades, le provocará vómitos, diarreas y deshidratación.
El trozozoito giardia lamblia es el parásito causante de la giardiasis. Este parásito se presenta con (+) en la línea base con un 100\%. Este porcentaje se distribuye en la línea de comparación entre $(+),(++)$ y $(+++)$ con $50 \%, 25 \%$ y $25 \%$, respectivamente. Lo que implica que la parasitosis se ha incrementado en la línea de comparación.
También existe una presencia del quiste endolimax nana, otro causante de la parasitosis, pero que resulta inofensivo siempre y cuando no se relacione con la histolytica o con la giardis. Sin embargo, en el caso de los niños en análisis es que en su gran mayoría presentan de manera conjunta. El quiste endolimax nana (+) representa el $77,4 \%$ en la línea base, pero aumenta a $84.7 \%$ en la línea de comparación. El nivel $(++)$ y $(+++)$ disminuyen con $16,1 \%$ y $6,5 \%$; respectivamente en la línea base. En la línea de comparación, el nivel $(++)$ se reduce a $15,4 \%$ mientras el nivel $(+++)$ desaparece por completo. Con ello, hasta el momento es el primero que se reduce de manera positiva.

Se manifiesta el quiste chilomastix mesnilli, protagonista de enfermedades gastrointestinales como la diarrea, únicamente en la línea de comparación. Para el nivel $(+)$ y $(++)$ se presenta en un $50 \%$ para cada uno. Por lo que, se sigue pretendiendo confirmar que la parasitosis es muy marcada en la línea de comparación.

Por otro lado, los piocitos se mostraron latentes en la línea base en sus dos niveles: $3-7$ y $22-27$ con un $50 \%$ cada uno. Sin embargo, en la línea de comparación desaparecen por completo. Este es un factor favorable debido a que implica la anulación de enfermedades cuyo origen sea bacteriano.

Un comportamiento similar se observó en los hematíes. En la línea base, se destacaron con $66,7 \%$ en el caso de $2-5$ campo, que es el más concentrado, y $33,3 \%$ cuando se trataba de 4-7 campo. Pero estos hematíes desaparecen en la línea de comparación. Es decir, hay una ausencia de enfermedades gastrointestinales severas. 
Tabla 3

Resultados de los exámenes coproparasitarios

\begin{tabular}{|c|c|c|c|}
\hline Resultado & Nivel & Línea base & Línea comparación \\
\hline \multirow[t]{2}{*}{ Moco } & + & $33,3 \%$ & $100,0 \%$ \\
\hline & ++ & $66,7 \%$ & $0,0 \%$ \\
\hline \multirow[t]{2}{*}{ Quiste ameba coli } & + & $97,4 \%$ & $66,7 \%$ \\
\hline & ++ & $2,6 \%$ & $33,3 \%$ \\
\hline \multirow{2}{*}{$\begin{array}{l}\text { Quiste ameba } \\
\text { histolytica }\end{array}$} & + & $100,0 \%$ & $78,6 \%$ \\
\hline & ++ & $0,0 \%$ & $21,4 \%$ \\
\hline Blastocystis hominis & + & $0,0 \%$ & $100,0 \%$ \\
\hline \multirow{3}{*}{$\begin{array}{l}\text { Trofozoito giardia } \\
\text { lamblia }\end{array}$} & + & $100,0 \%$ & $50,0 \%$ \\
\hline & ++ & $0,0 \%$ & $25,0 \%$ \\
\hline & +++ & $0,0 \%$ & $25,0 \%$ \\
\hline \multirow{3}{*}{$\begin{array}{l}\text { Quiste giardia } \\
\text { lamblia }\end{array}$} & + & $54,5 \%$ & $63,6 \%$ \\
\hline & ++ & $36,4 \%$ & $18,2 \%$ \\
\hline & +++ & $9,1 \%$ & $18,2 \%$ \\
\hline \multirow{3}{*}{$\begin{array}{l}\text { Quiste endolimax } \\
\text { nana }\end{array}$} & + & $77,4 \%$ & $84,6 \%$ \\
\hline & ++ & $16,1 \%$ & $15,4 \%$ \\
\hline & +++ & $6,5 \%$ & $0,0 \%$ \\
\hline \multirow{2}{*}{$\begin{array}{l}\text { Quiste chilomastix } \\
\text { mesnilli }\end{array}$} & + & $0,0 \%$ & $50,0 \%$ \\
\hline & ++ & $0,0 \%$ & $50,0 \%$ \\
\hline \multirow[t]{2}{*}{ Piocitos } & 3-7/campo & $50,0 \%$ & $0,0 \%$ \\
\hline & $\begin{array}{l}\text { 22-27/ } \\
\text { campo }\end{array}$ & $50,0 \%$ & $0,0 \%$ \\
\hline \multirow[t]{2}{*}{ Hematíes } & 2-5/campo & $66,7 \%$ & $0,0 \%$ \\
\hline & 4-7/campo & $33,3 \%$ & $0,0 \%$ \\
\hline
\end{tabular}

Fuente: Información de los exámenes coproparasitarios de la línea base del proyecto y del nuevo levantamiento de datos
En el caso de los controles médicos, para ver la eficacia de las actividades implantadas por el proyecto y visualizar al mediano plazo su incidencia en la población objetivo, se analizará en la presente sección los resultados de los exámenes coproparasitarios de línea base y de la línea de comparación. Para ello, se plantea la hipótesis nula en la que diferencia de la media entre la presencia o ausencia del microorganismo es cero en ambas líneas. Caso contrario, se toma en consideración la hipótesis nula en la que se menciona que la diferencia de medias del microorganismo al analizar las líneas es diferente de cero.

En la Tabla IV se puede evidenciar los principales causantes de la parasitosis en los niños en edad escolar de las escuelas 15 de Agosto y UNICEF. Dentro de los que se destacan los quistes: ameba coli, ameba histolytica, giardia lamblia y endolimax nana; así como, el trofozoíto giardia lamblia. De los cuales, solamente los quistes ameba coli y ameba histolytica son significativos al 95\%. Esto se puede concluir debido a que, la hipótesis nula resalta la no variación entre la línea base y la línea de comparación de los parásitos en análisis.

En cambio, la hipótesis nula relaciona este cambio por el tipo de año. Además, como los $\mathrm{p}$-valores son menores al nivel de significación (5\%), se rechaza la hipótesis nula y se acepta la hipótesis alternativa. Cabe mencionar que, a pesar de que dichos quistes no causen ninguna enfermedad por sí solos, son una evidencia contundente de la presencia de parasitosis en los niños del estudio. Sin embargo, es una situación que se podría mejorar con controles médicos frecuentes en los cuales se puede tomar asunto ante la aparición de estos microorganismos. 
Mejora de las condiciones higiénico-sanitarias, de la salud y ambientales de los niños en edad escolar de las comunidades Gatazo - Colta José I. López P., Jenny L. Basantes A. • VÍNCULOS-ESPE (2019) VOL. 4, No.3:41-55

\section{Tabla 4}

Diferencias significativas en los resultados de los exámenes coproparasitarios

\begin{tabular}{|l|r|r|r|}
\hline \multicolumn{5}{|c|}{ Pruebas de chi-cuadrado } \\
\hline \multicolumn{1}{|c|}{ Resultado } & Valor & gl & Sig. asintótica (bilateral) \\
\hline Quiste ameba coli & 11,654 & 1 & 0,001 \\
\hline Quiste ameba histolytica & 9,401 & 1 & 0,002 \\
\hline Trofozoito giardia lamblia & 0,833 & 2 & 0,659 \\
\hline Quiste giardia lamblia & 1,077 & 2 & 0,584 \\
\hline Quiste endolimax nana & 1,784 & 2 & 0,410 \\
\hline
\end{tabular}

Fuente: Información de los exámenes coproparasitarios de la línea base del proyecto y del nuevo levantamiento de datos

En el caso de los controles médicos, se aplicó un método cualitativo de evaluación. Se realizaron entrevistas con las autoridades encargadas en su momento de la participación en el proyecto de vinculación; así como, a los beneficiarios participantes. Las temáticas principales de la entrevista estaban direccionadas en tres ámbitos: conocimiento del proyecto, su implementación y los resultados alcanzados. Con ello, se evidenció que la presencia de parásitos está asociada con el consumo de agua debido a que el agua que llega a sus hogares no muestra características potables aptas para el consumo humano. Además, la mayor parte de las familias no cuenta con un seguro ni público ni privado que provea la atención para evitar trastornos básicos como la presencia de parásitos. También se enfatizó en que las charlas relacionadas a las buenas prácticas de higiene no fueron realizadas lo que también aportaría a la presencia de los microorganismos patógenos en el cuerpo de los infantes.

\section{CONCLUSIONES}

Los objetivos del proyecto de vinculación alcanzaron un cumplimiento del $55,56 \%$ puesto que las actividades planificadas fueron concluidas en esa medida; en tanto, el $27,78 \%$ de acciones a aplicarse se quedaron en proceso y el $16.67 \%$ no se llevaron a cabo.

En lugar de una disminución, se reflejó aumento en la proporción de niños con desnutrición del $1 \%$ al $8 \%$. Se demostró que la talla y el peso han variado significativamente al 95\%. De manera, similar se evidenció un cambio significativo en el estado nutricional. Por lo tanto, se puede afirmar que la desnutrición aumentó significativamente. Lo que implica, la anulación del cumplimiento del objetivo dos del proyecto de vinculación relacionado con la disminución de la desnutrición.

En cuanto a la alimentación adecuada se refleja un cambio significativo en el consumo de alimentos en la dieta diaria de la población objetivo. Incrementando el consumo de frutas y verduras. Adicionalmente, la variación de comidas en el día no ha sufrido un cambio. No obstante, debido a que las actividades de concientización y planteamiento de dietas saludables no fueron realizadas, no se puede concluir que el proyecto haya sido partícipe de este resultado.

Las actividades relacionadas con la concientización de la importancia de los controles médicos no fueron concluidas. Lo que implica, que los padres de familia no consideren relevante participar periódicamente en campañas de desparasitación. Tampoco asisten con frecuencia al médico debido a los costos que implican, especialmente en lo relacionado a la atención pues no poseen un seguro médico y la medicación les resulta excesivamente costosa. 
A pesar de que la evaluación era un objetivo del proyecto de vinculación no fue realizada; tampoco se hizo ningún tipo de monitoreo. Lo que implica un incumplimiento total de este objetivo.

Después de haber realizado algunos análisis de la información cuantitativa y cualitativa a través del método no experimental de evaluación de impacto y complementándolo con un método cualitativo como lo conforman las entrevistas, se puede concluir que el proyecto de vinculación con la sociedad ha alcanzado parcialmente los objetivos planteados.

\section{REFERENCIAS}

BAKER, J.L. (2000). Evaluación del impacto de los proyectos de desarrollo en la pobreza: manual para profesionales. Washington, D.C. Recuperado de http://documents.worldbank.org/curated/ en/974581468278042080/pdf/207450SPANISHOmanual.pdf

LEY NO. 298. (2010). Ley Orgánica de Educación Superior (LOES). Quito, Ecuador. 7 de octubre de 2010. Registro Oficial.

COHEN, E., \& MARTÍNEZ, R. (2010). Manual de formulación, evaluación y monitoreo de proyectos sociales. Comisión Económica para América Latina y el Caribe (CEPAL): División de Desarrollo Social. Recuperado de https://accionsocial.ucr.ac.cr/sites/default/files/ documentos/manual_formulacion.pdf

RODRÍGUEZ, U.V. (2007). Proyectos sociales y participación ciudadana. Visibilización del nuevo rol de la sociedad civil (Tesis de pregrado). Universidad de Chile, Santiago, Chile. Recuperado de http://repositorio. uchile.cl/tesis/uchile/2007/ubal_v/sources/ubal_v.pdf
GETLER, J., MARTíNEZ, S., PREMAND, P., RAWLINGS, L., \& VERMEERSCH, C. (2010). Impact Evaluation in Practice. Washington D.C. Banco Mundial.

ORGANIZACIÓN PARA LA COOPERACIÓN Y EL DESAROLLO ECONÓMICO (OCDE). (2010). Glosario de los principales términos sobre evaluación y gestión basada en resultados. París, Francia Recuperado de https://www.oecd.org/dac/evaluation/2754804. $\mathrm{pdf}$

COMISIÓN ECONÓMICA PARA AMÉRICA LATINA Y EL CARIBE (CEPAL). (2005). Manual para la evaluación de impacto de proyectos y programas de lucha contra la pobreza. Santiago de Chile.

COHEN, E., \& FRANCO, R. (1992). Evaluación de proyectos sociales. México.

NAVARRO, H. (2005). Manual para la evaluación de impacto de proyectos y programas de lucha contra la pobreza. Santiago de Chile, Chile. Instituto Latinoamericano y del Caribe de Planificación Económica y Social (ILPES).

HERNÁNDEZ, R., FERNÁNDEZ, C. \& BAPTISTA, L. (2014). Metodología de la Investigación. México: McGraw Hill.

RODRíGUEZ, A. Y PÉREZ, A. O. (2017). Métodos científicos de indagación y de construcción del conocimiento Revista EAN, 82. https://doi.org/10.21158/01208160.n82.2017.1647.

GALINDO, E. (2005). Estadística: métodos y aplicaciones. Quito, Ecuador: ProCiencia Editores. 